\title{
Upper Vaginectomy
}

National Cancer Institute

\section{Source}

National Cancer Institute. Upper Vaginectomy. NCI Thesaurus. Code C136237.

The surgical removal of the upper part of the vagina. 